\title{
On Symmetric Water Waves with Constant Vorticity
}

Florian Kogelbauer

To cite this article: Florian Kogelbauer (2015) On Symmetric Water Waves with Constant Vorticity, Journal of Nonlinear Mathematical Physics 22:4, 494-498, DOI: https://doi.org/10.1080/14029251.2015.1113044

To link to this article: https://doi.org/10.1080/14029251.2015.1113044

Published online: 04 January 2021 


\title{
On Symmetric Water Waves with Constant Vorticity
}

\author{
Florian Kogelbauer \\ Faculty of Mathematics, University of Vienna, Oskar-Morgenstern-Platz 1 \\ Vienna, A-1090, Austria \\ florian.kogelbauer@univie.ac.at
}

Received 1 August 2015

Accepted 2 October 2015

\begin{abstract}
We prove that a solution to the gravity water wave problem with constant vorticity, whose wave profile as well as its horizontal velocity component at the free surface are symmetric at any instant of time, is given by a traveling wave. The proof is based on maximum principles and structural properties of the governing equations.
\end{abstract}

Keywords: Travelling waves; constant vorticity; symmetry.

2000 Mathematics Subject Classification: 35Q31, 42B37, 76B15

\section{Introduction}

Symmetry is ubiquitous for two-dimensional traveling waves in the absence of underlying currents $[11,17,20]$. It turns out that if the free surface is monotone between successive crests and troughs, then the wave is always symmetric - this holds true as well for waves with underlying currents, at least in the absence of stagnation points [2,3].

While water flows that originate in the state of irrotational flow are irrotational everywhere at all times thereafter (see [1]), non-uniform currents often exist before the waves are generated and in this case their effect has to be taken into consideration, cf. $[18,19]$. The investigation of wave-current interactions is quite intricate (experimentally, as well as theoretically), but, within the framework of two-dimensional flows, the vorticity of a particle is preserved as the particle moves about (see [1]) and this permits us to classify in such a setting wave-current interactions in terms of the vorticity distribution of the flow. In particular, non-zero vorticity is the hallmark of non-uniform underlying currents. For rotational water waves, the qualitative properties of the underlying flow can be described to good accuracy by considering the mean vorticity instead of the precise vorticity distribution, cf. $[9,13]$. This observation and the importance of vorticity in the modeling of ocean flows (since near-surface ocean currents are primarily caused by the wind - see e.g. the discussion in [4]) naturally leads to the study of traveling waves in flows with constant vorticity (see $[2,3,6]$ ). Moreover, the most regular and significant currents on areas of the continental shelf are the tidal currents, and the induced shear is usually viewed as having constant vorticity [5]: positive for the ebb current (associated with a falling tide) and negative for the flood current (associated with a rising tide) - the tidal currents being the alternating horizontal movements of water associated with the rise and fall of the tide. Note that a non-zero constant vorticity induces new dynamical features with respect to the case of irrotational flows, e.g. the possible appearance of Kelvin cat's eyes - see the discussion in $[8,21]$. For recent numerical simulations of the flow pattern in a water wave with 
constant vorticity we refer to $[5,14,15]$, while theoretical investigations of waves of large amplitude in flows with constant vorticity are pursued in [7].

In the following we will show that the symmetry results for irrotational water waves obtained recently in [16] can be adapted to the case of constant vorticity. The key to this is the fact that each component of the velocity field is harmonic, even if they are not harmonic conjugates of each other.

\section{The governing equations}

The governing equations for an ideal, incompressible, homogeneous fluid in two spatial dimensions in the presence of gravity are given by Euler's equations

$$
\left.\begin{array}{l}
u_{t}+u u_{x}+v u_{y}=-P_{x}, \\
v_{t}+u v_{x}+v v_{y}=-P_{y}-g
\end{array}\right\}
$$

and the equation of mass conservation

$$
u_{x}+v_{y}=0
$$

where $(u, v)=(u(x, y, t), v(x, y, t))$ is the velocity field, $P=P(x, y, t)$ is the pressure and $g \approx 9.81$ $\mathrm{m} / \mathrm{s}^{2}$ is the acceleration of gravity at the surface of the Earth. We assume that the fluid cannot penetrate a flat bed, hence

$$
v=0 \quad \text { on } \quad y=-d,
$$

where $d$ is the mean depth. The mathematically convenient assumption of a flat, horizontal bed is motivated practically by waves propagating in a canal but also by waves at the surface of the sea, since abyssal plains - the flattest areas on Earth, with an almost total absence of geographic features - are found in all major sea and ocean basins, covering overall almost a third of the Earth's surface (as much as all land above water). Modeling a free surface flow, we additionally require that the motion of the flow is decoupled from the motion of the air above, since the density of water is about $10^{3}$ times greater than that of the air. This is reflected by the requirement that the pressure is constant at the free surface

$$
P=P_{\text {atm }} \quad \text { on } \quad y=\eta(x, t),
$$

where $P_{\text {atm }}$ is the (constant) atmospheric pressure, while $y=\eta(x, t)$ is the graph of the free surface; moreover, the constraint

$$
v=\eta_{t}+u \eta_{x} \text { for } \quad y=\eta(x, t),
$$

should hold - this kinematic boundary condition expresses the fact that the free surface is an interface (particles on it are confined to it). In the following, we are dealing with flows of constant vorticity, which is expressed by

$$
u_{y}-v_{x}=\gamma
$$

for some constant $\gamma \in \mathbb{R}$. Equations (2.1-2.6) constitute the governing equations for the water wave problem with constant vorticity. For a detailed derivation and physical background of the above 
equations, we refer to $[1,12]$. The functions $u, v, P$ and $\eta$ are assumed to be smooth and $L$-periodic in the $x$-variable. At any instant of time, the unknown fluid domain is given by

$$
\Omega=\Omega(t)=\left\{(x, y) \in \mathbb{R}^{2}: 0<x<L,-d<y<\eta(x, t)\right\} .
$$

\section{Main Result}

The stream function $\psi(x, y, t)$ is defined as a solution to the system

$$
\left.\begin{array}{l}
\psi_{y}(x, y, t)=u(x, y, t), \\
\psi_{x}(x, y, t)=-v(x, y, t),
\end{array}\right\}
$$

at any instant of time. With a look at (2.2) it may be written explicitly as

$$
\psi(x, y, t)=\int_{\left(x_{0}, y_{0}\right)}^{(x, y)} u d y-v d x
$$

where integral has to be taken along any path in the fluid domain joining some fixed point $\left(x_{0}, y_{0}\right)$ and $(x, y)$. The function $\psi$ is $L$-periodic in the $x$-variable and satisfies Poisson's equation

$$
\Delta \psi=\gamma
$$

in the fluid domain $\Omega$.

Theorem 3.1. Let $(u, v, P, \eta)$ be an x-periodic solution to the water wave problem with constant vorticity (2.1)-(2.6) with the property that the free surface $\eta$ as well as the horizontal component $u$ at the surface are both symmetric about an axis of symmetry $x=\lambda(t)$ at any instant of time. Then the solution defines a traveling wave.

The first assumption reads as

$$
\eta(x, t)=\eta(2 \lambda(t)-x, t)
$$

at any instant of time, while the second assumption reads as

$$
u(x, \eta(x, t), t)=u(2 \lambda(t)-x, \eta(x, t), t)
$$

at any instant of time. We will use the following lemma, derived in [10], to prove that the flow is that of a traveling wave.

Lemma 3.1. Only traveling wave solutions solution to the governing equations (2.1)-(2.6) have the property that

$$
\begin{gathered}
u(x, y, t)=u(2 \lambda(t)-x, y, t), \\
v(x, y, t)=-v(2 \lambda(t)-x, y, t), \\
P(x, y, t)=P(2 \lambda(t)-x, y, t), \\
\eta(x, t)=\eta(2 \lambda(t)-x, t)
\end{gathered}
$$

throughout the fluid domain. 
Proof. We will show by maximum principles that the function

$$
\Psi(x, y, t):=\psi(x, y, t)-\psi(2 \lambda(t)-x, y, t),
$$

which is $L$-periodic, harmonic by (2.6) and zero at the flat bed by (2.3), is identically zero in $\Omega$. If both the maximum and the minimum would be attained at the flat bed, where $\Psi=0$ by (2.3), the function $\Psi$ would already be identically zero. So assume without loss of generality that the maximum of $\Psi$ is attained at the free surface. The tangential derivative of $\Psi$ at the maximum is zero,

$$
\frac{d}{d x} \Psi(x, \eta(x, t), t)=-v(x, \eta, t)-v(2 \lambda-x, \eta, t)+\eta_{x}(u(x, \eta, t)-u(2 \lambda-x, \eta, t))=0,
$$

in view of (3.1), which implies with assumption (3.5) that

$$
v(x, \eta, t)=-v(2 \lambda-x, \eta, t)
$$

at the maximum. Hopf's Lemma implies that the outward normal derivative at a maximum would be strictly positive, that is to say

$$
\eta_{x}(v(x, \eta, t)+v(2 \lambda-x, \eta, t))+u(x, \eta, t)-u(2 \lambda-x, \eta, t)>0,
$$

which is clearly a contradiction in view of (3.5) and (3.12). We infer that $\psi(x, y, t)=\psi(2 \lambda-x, y, t)$ for all $(x, y) \in \Omega$ and hence by (3.1)

$$
\begin{gathered}
u(x, y, t)=u(2 c(t)-x, y, t) \\
v(x, y, t)=-v(2 c(t)-x, y, t)
\end{gathered}
$$

throughout the fluid.

Applying Hopf's maximum principle to the function $\tilde{P}(x, y, t):=P(x, y, t)-P(2 \lambda(t)-x, y, t)$, which is harmonic in view of (2.1), (2.2) and (3.14), at the flat bed in combination with the boundary condition (2.3) and the second Euler equation in (2.1), we deduce that $\tilde{P}(x, y, t) \equiv 0$ in $\Omega$ and therefore that the pressure is symmetric. By lemma (3.1), the flow defines a traveling wave.

\section{Acknowledgments}

The financial support of the ERC Advanced Grant "Nonlinear Studies of water flows with vorticity" (NWFV 267116) is acknowledged.

\section{References}

[1] A. Constantin. Nonlinear water waves with applications to wave-current interactions and tsunamis. CBMS-NSF Regional Conference Series in Applied Mathematics. SIAM, 2011.

[2] A. Constantin, M. Ehrnström, and E. Wahlén. Symmetry of steady periodic gravity water waves with vorticity. Duke Math. J., 140(3):591-603, 2007.

[3] A. Constantin and J. Escher. Symmetry of steady periodic surface water waves with vorticity. J. Fluid Mech., 498:171-181, 2004.

[4] A. Constantin and R. S. Johnson. The dynamics of waves interacting with the equatorial undercurrent. Geophys. Astrophys. Fluid Dyn., 109(4):311-358, 2015.

[5] A. Constantin, K. Kalimeris, and O. Scherzer. A penalization method for calculating the flow beneath traveling water waves of large amplitude. SIAM J. Appl. Math., 75:1513-1535, 2015. 
[6] A. Constantin and W. Strauss. Exact steady periodic water waves with vorticity. Comm. Pure Appl. Math., 57:481-527, 2004.

[7] A. Constantin, W. Strauss, and E. Varvaruca. Global bifurcation of steady gravity water waves with critical layers. arXiv:1407.0092, 2014.

[8] A. Constantin and E. Varvaruca. Steady periodic water waves with constant vorticity: Regularity and local bifurcation. Arch. Rat. Mech. Anal., 199(1):33-67, 2010.

[9] A. F. T. Da Silva and D. H. Peregrine. Steep, steady surface waves on water of finite depth with constant vorticity. J. Fluid Mech., 195:281-302, 101988.

[10] M. Ehrnström, H. Holden, and X. Raynaud. Symmetric Waves Are Traveling Waves. International Mathematics Research Notices, 2009(24):4578-4596, 2009.

[11] P. R. Garabedian. Surface waves of finite depth. J. Analyse Math., 14:161-169, 1965.

[12] R. S. Johnson. A Modern Introduction to the Mathematical Theory of Water Waves. Cambridge Texts in Applied Mathematics, Cambridge, 1997.

[13] I. G. Jonsson. Wave-current interactions. In B. Mehaute and D. M. Hanes, editors, The Sea, pages 65-120. Wiley, New York, 1990.

[14] J. Ko and W. Strauss. Effect of vorticity on steady water waves. J. Fluid Mech., 608:197-215, 2008.

[15] J. Ko and W. Strauss. Large-amplitude steady rotational water waves. Eur. J. Mech. B Fluids, 27:96109, 2008.

[16] F. Kogelbauer. Symmetric irrotational water waves are traveling waves. J. Diff. Eq., 259(10):5271-5275, 2015.

[17] H. Okamoto and M. Shoji. The Mathematical Theory of Permanent Progressive Water-waves. World Scientific, 2001.

[18] G. P. Thomas. Wave-current interactions: an experimental and numerical study. J. Fluid Mech., 216:505-536, 1990.

[19] G. P. Thomas and G. Klopman. Wave-current interactions in the nearshore region. In J. N. Hunt, editor, Gravity Waves in Water of Finite Depth, volume 10, pages 215-319. Computational Mechanics Pbulications, 1997.

[20] J. F. Toland. Stokes waves. Topol. Methods Nonlinear Anal., 7(1):1-48, 1996.

[21] E. Wahlén. Steady water waves with a critical layer. J. Diff. Eq., 246(6):2468-2483, 2009. 